\section{Cone- and rod- mediated multifocal electroretinogram in early age-related maculopathy}

\author{
B Feigl ${ }^{1,2}$, B Brown², J Lovie-Kitchin² and P Swann²
}

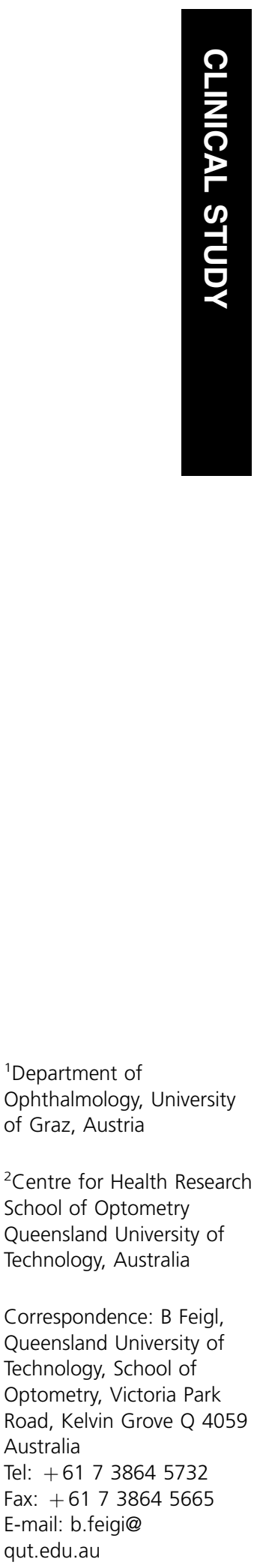

\begin{abstract}
Purpose To investigate the cone- and rodmediated multifocal electroretinograms (mfERG) in early age-related maculopathy (early ARM).

Methods and subjects We investigated the cone- and rod-mediated mfERG in 17 eyes of 17 subjects with early ARM and 16 eyes of 16 age-matched control subjects with normal fundi. All subjects had a visual acuity of 6/12 or better. We divided the ARM subjects into two groups based on drusen size and retinal pigment epithelium abnormalities - a less advanced (ARM1) and a more advanced (ARM2) group. The mfERG data were compared to templates derived from the control group. We analysed the mfERG results for the central and peripheral fields (CP method) and the superior and inferior fields (SI method).
\end{abstract}

Results While the mean cone results showed no statistically significant difference between the groups, the rods showed significantly delayed responses in the ARM1 group for the CP and the SI methods, but not in the ARM2 group, although there was a trend of longer latencies compared to the control group. Conclusion Our results show a functional impairment of the rods in early ARM subjects. As there is histopathological evidence showing earlier rod than cone impairment in early ARM, following the rod function with the mfERG might be helpful in diagnosis or for monitoring the progression of early ARM.

Eye (2005) 19, 431-441. doi:10.1038/sj.eye.6701503 Published online 30 July 2004

Keywords: multifocal electroretinogram; MfERG; cones; rods; early age-related maculopathy; early ARM

\section{Introduction}

Recent histopathological studies by Curcio et al. ${ }^{1-4}$ have shown a preferential vulnerability of rods compared to cones in age-related maculopathy (ARM) eyes. They found no difference between normal age-matched eyes and early ARM eyes in the foveal mosaic, but parafoveally there was a localized rod loss together with mis-shapen cone inner segments in early ARM eyes. Furthermore, they demonstrated that the rod loss began in the inferior retina during normal ageing. Earlier histopathologic studies in ageing eyes also indicated preferential rod damage with elongation and nuclear displacement starting at age 40 years. ${ }^{5,6}$ Holopigian et al. ${ }^{7}$ investigated the peripheral cone and rod function in subjects with early ARM by obtaining dark-adaptation curves, electro-oculograms (EOG), and full-field electroretinograms (ERG). They found no difference for the cones compared to agematched controls but abnormal absolute thresholds and cone-rod break times as well as impaired electroretinographic measures in the full-field ERG for the rod system. Owsley et al. ${ }^{8,9}$ measured scotopic sensitivity at 52 loci in the central $38^{\circ}$ field and showed delayed rodmediated dark adaptation in early ARM. Sunness et al. ${ }^{10}$ tried to differentiate between ARM stages by performing focal EOGs but found that the focal EOG was not sensitive enough to discriminate between ARM subgroups. Recently, Scholl et al. ${ }^{11}$ showed normal or mildly abnormal photopic sensitivity, but moderately to severely reduced scotopic sensitivity in ARM subjects with central increased fundus autofluorescence corresponding to foveal drusen. They performed fine matrix mapping with a modified Humphrey field analyser depending on the location of the area of increased fundus
Ophthalmology, University of Graz, Austria

${ }^{2}$ Centre for Health Research School of Optometry Queensland University of Technology, Australia

Correspondence: B Feigl Queensland University of Australia

Tel: +61 738645732

Fax: +61 73864566

qut.edu.au

Received: 22 October 2003 Accepted: 2 February 2004 Published online: 30 July 2004 
autofluorescence imaged with a confocal scanning laser ophthalmoscope. Curcio et $a l .^{3}$ suggested that tests of rod function might be advantageous, and might permit detection of ARM at earlier stages than do standard tests of cone function.

The multifocal electroretinogram (mfERG) developed by Sutter and Tran ${ }^{12}$ can give valuable information about the functional condition of the central retina. ${ }^{13-17}$ Most previous studies have been performed under photopic conditions, which are thought to reflect mainly conemediated photoreceptor and bipolar function. ${ }^{18,19}$

Although reduced amplitudes and delayed latency of the cone mfERG have been demonstrated with age $\mathrm{e}^{20-22}$ and in different stages of $\mathrm{ARM}^{23-26}$ mainly with concentric ring averaging methods, there have been no studies identifying the functional impairment of rods. Recently Hood et al. ${ }^{27}$ developed a mfERG protocol for isolating rod responses in humans and Holopigian et al..$^{28,29}$ demonstrated the clinical use of this protocol in retinitis pigmentosa and progressive cone dystrophy.

Based on these histological and psychophysical results, our main goal was to measure the function of both cones and rods in different advanced ARM groups, using the mfERG and a curve fitting method for objectively characterizing $\mathrm{mfERG}$ parameters.

\section{Materials and methods}

\section{Subjects}

In all, 33 subjects were selected from the Optometry Clinic at the Queensland University of Technology, Brisbane, Australia or were referred by local ophthalmologists. Of these, 17 subjects made up the early ARM group (nine female and eight male subjects, age range 60-75 years, mean age 70 years) and 16 subjects comprised the age-matched control group (eight female and eight male subjects, age range 58-72 years, mean age 68 years). All subjects had distance visual acuity of $6 / 12$ or better. They underwent a full clinical examination, including slit-lamp and fundus photography and if necessary fluorescein angiography to exclude choroidal neovascularization. All subjects who were enrolled in the study gave written informed consent, and the tenets of the Declaration of Helsinki and the requirements of the University Human Research Ethics Committee of the Queensland University of Technology were followed.

Slit-lamp photographs for grading the lens were taken and subjects with posterior subcapsular cataract and cortical or nuclear opacities higher than grade one according to the templates of the Age-Related Eye Disease Study (AREDS) clinical lens standards were excluded..$^{30}$ Colour fundus photographs (Zeiss Jena Mydriatic Fundus Camera) of the central $30^{\circ}$ of the posterior pole (centred on the fovea) were taken and retinal changes were graded independently by two experienced observers (BF, PS) by using a set of the Wisconsin age-related maculopathy standards with example photographs and a grid with grading circles (AREDS Reading Center, University of Wisconsin, Madison, USA). ${ }^{31}$ One observer (PS) was masked to the subjects' functional results, and agreement was achieved in all subjects with judgments of the other investigator (BF, not masked).

We defined early ARM as the presence of either hard or soft distinct drusen less than $125 \mu \mathrm{m}$ in size with or without retinal pigment epithelial (RPE) abnormalities or soft indistinct drusen larger than $125 \mu \mathrm{m}$. Both types of drusen have been shown to damage cone and rod inner and outer segments. ${ }^{32}$ We divided our subjects into two groups (ARM1 and ARM2) according to the AREDS AMD levels ${ }^{31}$ (ARM1 = AREDS ARM levels 1 and 2; ARM2 = AREDS ARM level IIIa-c) (Table 1). The ARM1 group (less advanced) consisted of 11 eyes with drusen size less than $125 \mu \mathrm{m}$ and RPE abnormalities, and the ARM2 group (more advanced) included six eyes with indistinct drusen greater than $125 \mu \mathrm{m}$ and/or a total area $372 \mu \mathrm{m}$ determined 'by mentally moving all drusen together' ${ }^{31}$ and comparing this area with those of the standard circles of the Wisconsin grading system. These two groups represent an increasing risk to develop late ARM. ${ }^{33}$ The control subjects ( $\mathrm{N}$ group), with visual

Table 1 ARM subjects divided into two groups (ARM1 and ARM2) according to the AREDS ARM main levels

Groups

\section{ARM 1}

Drusen size $(\mu m)(L I)$

$<63 \mu \mathrm{m}$ and total area $<125 \mu \mathrm{m}$

Presence of one or more of the following (LII)

(a) Drusen max size $\geqslant 63 \mu \mathrm{m}$ but $<125 \mu \mathrm{m}$

(b) Drusen total area $\geqslant 125 \mu \mathrm{m}$

(c) Retinal pigment epithelial abnormalities consistent with AMD, defined as one or more of the following in the central or inner subfield:

(1) Depigmentation present

(2) Increased pigment $\geqslant 125 \mu \mathrm{m}$

(3) Increased pigment present and depigmentation at least questionable

\section{ARM 2}

Presence of one of the following (LIII)

(a) Drusen max size $\geqslant 125 \mu \mathrm{m}$

(b) Drusen max size $\geqslant 63 \mu \mathrm{m}$ and total area $>372 \mu \mathrm{m}$, type is soft indistinct

(c) Drusen max size $\geqslant 63 \mu \mathrm{m}$ and $>$ total area $650 \mu \mathrm{m}$ and type is soft distinct

(d) Geographic atrophy within grid but none at the centre of macula 
acuities of $6 / 6$ or better and normal fundi, made up the third group (16 eyes). Table 2 shows the visual acuities and grading results for each of the ARM subjects.

\section{mfERGs}

The mfERGs (VERIS I, EDI Inc., San Mateo, CA, USA) were recorded monocularly using DTL thread electrodes and optical correction for the stimulus viewing distance $(50 \mathrm{~cm})$. Pupils were maximally dilated with tropicamide $0.5 \%$ and phenylephrine $2.5 \%$. The subjects were instructed to watch the centre of a monitor flickering between black and white hexagons. To help to maintain fixation, a cross extending to each corner of the screen was used. The stimulus for the cones was a 103 hexagon array $\left(35.5^{\circ} \times 28^{\circ}\right)$ and for the rods was a 61 hexagon array $\left(33^{\circ} \times 28^{\circ}\right)$. Retinal signals were bandpass filtered $(1-300 \mathrm{~Hz})$ and amplified (Grass P5 amplifier, $\times 100000)$; blinks or small eye movements causing ERG artefacts during the recording segments were detected online and those segments were rejected and re-recorded. Fixation was controlled by observing the subjects' recordings online on a monitor and by analysing the region of the blind spot after the recordings. An overlap test for both protocols was performed to exclude kernel overlap.

\section{Cone-mediated mfERG}

The frame rate of the 103 hexagon display was $67 \mathrm{~Hz}$, and the hexagons flickered according to a pseudorandom binary $\mathrm{m}$-sequence $\left(2^{13}-1\right.$ steps in length), with a luminance of $100 \mathrm{~cd} / \mathrm{m}^{2}$ for the white hexagons and $2 \mathrm{~cd} / \mathrm{m}^{2}$ for the black hexagons (measured with a Topcon BM-7 luminance colorimeter). Recordings were divided

Table 2 Grading result for each subjects' right (re) or left eye (le) and the distance visual acuity (VA)

\begin{tabular}{llll}
\hline Subjects & Eye & \multicolumn{1}{c}{ VA } & Group \\
\hline $\mathrm{a}$ & re & $6 / 6$ & ARM1 \\
$\mathrm{b}$ & re & $6 / 12$ & ARM2 \\
$\mathrm{c}$ & re & $6 / 6$ & ARM1 \\
$\mathrm{d}$ & re & $6 / 12$ & ARM1 \\
$\mathrm{e}$ & re & $6 / 6^{-1}$ & ARM1 \\
$\mathrm{f}$ & re & $6 / 7.5$ & ARM2 \\
$\mathrm{g}$ & re & $6 / 12$ & ARM1 \\
$\mathrm{h}$ & re & $6 / 7.5$ & ARM1 \\
$\mathrm{i}$ & re & $6 / 7.5$ & ARM2 \\
$\mathrm{j}$ & re & $6 / 7.5$ & ARM1 \\
$\mathrm{k}$ & re & $6 / 9.5^{+1}$ & ARM2 \\
$\mathrm{l}$ & re & $6 / 9.5$ & ARM2 \\
$\mathrm{m}$ & le & $6 / 6^{-1}$ & ARM2 \\
$\mathrm{n}$ & le & $6 / 6^{-2}$ & ARM1 \\
$\mathrm{o}$ & le & $6 / 6^{-1}$ & ARM1 \\
$\mathrm{p}$ & re & $6 / 9.5^{+1}$ & ARM1 \\
$\mathrm{q}$ & re & $6 / 9.5^{-2}$ & ARM1 \\
\hline
\end{tabular}

into 16 segments (each about $11 \mathrm{~s}$ ). Four files for averaging were obtained from every subject, resulting in a total recording time of about $10 \mathrm{~min}$ per eye (not including resting time between the segments). Figure 1a shows the hexagonal arrays for the cones with the trace arrays of a control subject below.

\section{Rod-mediated mfERG}

Rod-mediated mfERGs were recorded by applying a method similar to that of Hood et al. ${ }^{27}$ We used a blue Wratten 47B filter (W47B) and slowed the stimulus sequence by inserting three blank frames between stimulus frames. We performed control experiments to find the optimal stimulus luminance and surround conditions to minimize stray light influence. ${ }^{27} \mathrm{We}$ performed several stimulus luminance experiments by varying the neutral density (ND) filters (W47B alone, $\mathrm{W} 47 \mathrm{~B}+1.0 \mathrm{ND}$, and W47B + 1.5 ND) with no added surround and maximal ND filter setup (W47B + 1.5 ND) with surround. Figure 2 shows the summed overall unfiltered rod responses to different surround luminances $\left(0.01,0.009,0.004 \mathrm{~cd} / \mathrm{m}^{2}\right)$ demonstrating decreased amplitudes with increased surround luminance. With no surround $\left(0.0005 \mathrm{~cd} / \mathrm{m}^{2}\right)$, a large stray light response was found.

For our protocol we chose the lowest light condition and the blue filter $\left(\mathrm{W} 47 \mathrm{~B}+1.5 \mathrm{ND}, 0.004 \mathrm{~cd} / \mathrm{m}^{2}\right.$ surround), which gave us the highest rod response. Thus, the luminance levels for our rod protocol were

a

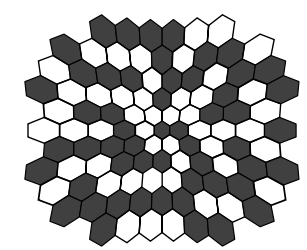

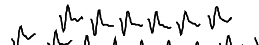

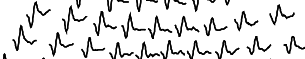

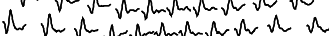

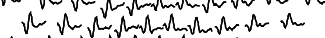

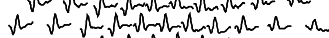

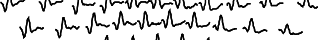

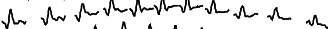

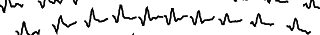
undunanan $50 \mathrm{nV}$ L undurand $100 \mathrm{~ms}$ b

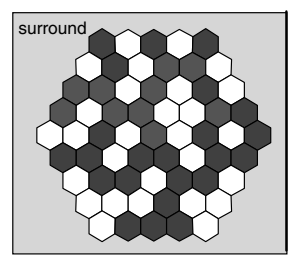

Figure 1 mfERG stimulus array (top) with (a) 103 scaled hexagons for the cones and (b) 61 unscaled hexagons and a surround for the rods and the filtered normal responses from a trained control subject (lower panel) right eye. Note the different scaling for the rod-mediated traces and the lower responses nasally indicating the region of the blind spot. 


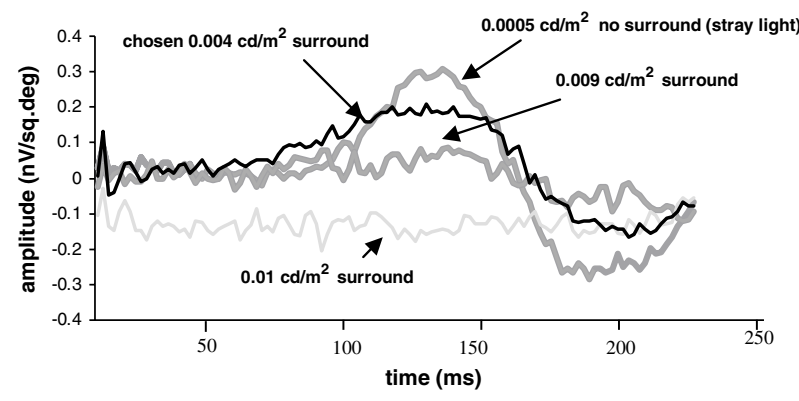

Figure 2 Unfiltered rod-mediated mfERG overall response for a trained subject for different surround conditions (no surround $=0.0005,0.004,0.009,0.01 \mathrm{~cd} / \mathrm{m}^{2}$ ). A large stray light response was found without a surround and an extinguished response with a bright surround $\left(0.01 \mathrm{~cd} / \mathrm{m}^{2}\right)$ was evident. Note that the raw overall amplitude values are shown in $\mathrm{nV} / \mathrm{sq}$ deg (absolute amplitude divided by the area from which the response was evoked).

$0.0098 \mathrm{~cd} / \mathrm{m}^{2}$ for the bright, $0.0005 \mathrm{~cd} / \mathrm{m}^{2}$ for the $\mathrm{dim}$ hexagons, and $0.004 \mathrm{~cd} / \mathrm{m}^{2}$ for the surround (calculated from measurements made with a Topcon BM-7 luminance colorimeter).

After 40 min of dark adaptation, the rod mfERGs were recorded with a stimulus array of 61 equally sized hexagons, which flickered concurrently according to a pseudorandom binary m-sequence ( $2^{13}-1$ steps in length). One recording was obtained for each subject; it was divided into 32 segments (each lasting about $21 \mathrm{~s}$ ), resulting in a total recording time of about $11 \mathrm{~min}$ (not including resting time between the segments). Figure $1 \mathrm{~b}$ shows the hexagonal array for the rods and a trace array of a young and trained control observer. A different scaling for the rod traces was chosen to point out the different and smaller waveforms compared to the cones. The rod-mediated waveforms are much smaller and broader and show a later, less distinct peak compared to the cone-mediated response. ${ }^{27}$ Although local (foveal and optical disc) responses are difficult to obtain, ${ }^{27}$ the reduced nasal responses in Figure $1 \mathrm{~b}$ possibly indicate the blind spot area. As with the cone-mediated mfERG, ${ }^{34}$ the blind spot area was not evident in every subject and a response could be obtained in this area due to hexagon size and overlap of the stimulus elements.

\section{Analysis}

We examined the first-order kernel ERG response, which is the mean local response to all the flashes occurring in a stimulus cycle. ${ }^{35,36}$ Currently, there are three methods available for analysing the mfERG data. The conventional peak-to-peak (N1P1) and implicit time (N1latency, P1-latency) method, the scalar product method, ${ }^{12,37}$ and a newer method using a least-squares fitting procedure developed by Hood and $\mathrm{Li}^{38}$ We applied Hood and Li's ${ }^{38}$ method, which has proven to be valuable in hereditary retinal diseases, diabetes, and in establishing normative data for the mfERG. ${ }^{21,28,29,39} \mathrm{We}$ chose this curve-fitting procedure (Matlab, Mathworks, Natick, MA, USA) to detect slight functional deficits in our early ARM subjects who had relatively good visual acuity. Further, we thought the conventional peak-topeak amplitude and implicit time method to be less accurate for the rod results as the rod functions show no distinct peak and are broad, small and low-frequency potentials (Figure 1b). ${ }^{27}$ We derived normal templates for each of the 103 and 61 hexagons from the control subjects. We then fitted each ARM subjects' results to those by varying two parameters. One parameter scaled the amplitude (a-scale) at each point in the hexagon array to minimize the difference between the response and the template values and the other parameter was a multiplicative scaling of time ( $\mathrm{t}$-scale) that stretches the entire response waveform. For each hexagon, a leastsquares fitting procedure was used to find the best fit. For each record, two values were available: an amplitude (ascale) and a latency (t-scale) value, which indicated values relative to the normal template. In addition, an error statistic (statfit) described the quality of the fit to which both the a- and t-scale contributed. A perfect fit or stafit value of 0.0 is the result of an a- and t-scale value of 1.0, which indicates that the values are equal to the mean of a control group. For example an a-scale of 0.8 indicates that the response is $80 \%$ of that of the mean of control group value. Higher t-scale values such as 1.2 indicate $20 \%$ longer latencies than the mean of the control template. $^{38}$

Before fitting the cone and rod responses, the data were spatially averaged once (ratio $=6)$ and the VERIS noise reduction procedure (NRP) was performed. ${ }^{12}$ Signals were low-pass filtered at $80 \mathrm{~Hz}$ (cones) and $15 \mathrm{~Hz}$ (rods) resulting in better measurable signals for the fitting method without appreciable amplitude loss; cone and rod data were fitted over $45 \mathrm{~ms}$ (from 15 to $60 \mathrm{~ms}$ ) and $50 \mathrm{~ms}$ (from $60 \mathrm{~ms}$ to $110 \mathrm{~ms}$ ), respectively, to avoid early and late transients.

To determine which parameter (a- or t-scale value) best describes early ARM, we analysed the results by averaging the a- and $t$-scale results for the central (acen, tcent) and the peripheral fields (aperi, tperi) (CP method) and the superior (asup, tsup) and the inferior fields (ainf, tinf) (SI method), which correspond to the lower and upper retina, respectively, in our normal and ARM subjects (Figure 3).

We chose the CP and SI methods for the cones and rods because of histopathological evidence of preferential rod vulnerability beginning in the lower retina and parafoveally during ageing and in early ARM, and a hypothesized cone-rod interaction previously suggested 


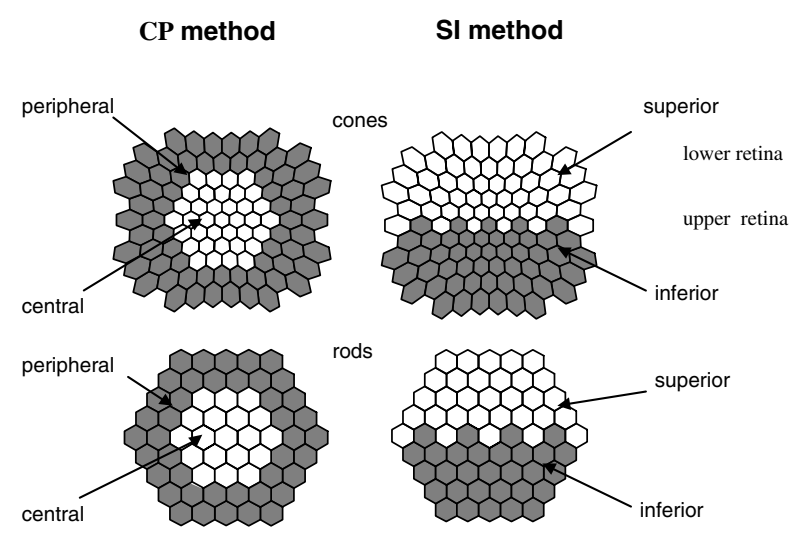

Figure 3 In the CP method, we analysed the mean results from the central hexagon and the surrounding three rings for the cones and the central hexagon and the surrounding two rings for the rods and compared those with the means from the outer peripheral rings. The SI method compared the mean results from the superior (lower retina) and inferior (upper retina) fields.

by Hicks et al..$^{40}$ The reason for not excluding the central hexagon, which is supposed to be a rod-free area, was the relatively large hexagon size we chose (about $3^{\circ}$ ), which probably included some rod responses. Additionally, Hood et al. ${ }^{27}$ discussed the difficulty of obtaining local rod responses even with optimal recordings and found it hard to discern the fovea and the optic disc. Choosing smaller hexagons for more precise local rod distributions ${ }^{27}$ would have resulted in a poorer signal-tonoise ratio and would have required longer recording sessions, which in our older subjects we thought was too demanding.

The mean statfit value for cones $(0.3 \pm 0.1$ for all fields $)$ and for rods $(0.3 \pm 0.1$ for the central and inferior field and $0.4 \pm 0.1$ for the peripheral and superior fields) showed low values, indicating that the template fits were accurate over the whole field.

Cone-mediated mfERGs were recorded from 17 ARM eyes and from 16 control eyes.

Rod-mediated mfERGs were recorded from 14 ARM eyes and 13 control eyes (ARM subjects $1, p, q$, and three control subjects could not perform the rod mfERG procedure). Recent studies suggested using a quality (goodness) of the fit ${ }^{28,29,38}$ criterion for deciding which records are responses and which are noise. After examining our data, we set a statfit value of lower than 0.8 to identify a response from noise (Figure 4 ). Results higher than 0.8 were considered as noise. The records of the left eyes were mirror imaged so that appropriate parts of the retina were being compared across eyes.

\section{Statistical analysis}

Data were analysed with the Statistical Package for the Social Sciences (SPSS-11). Repeated measures analysis of

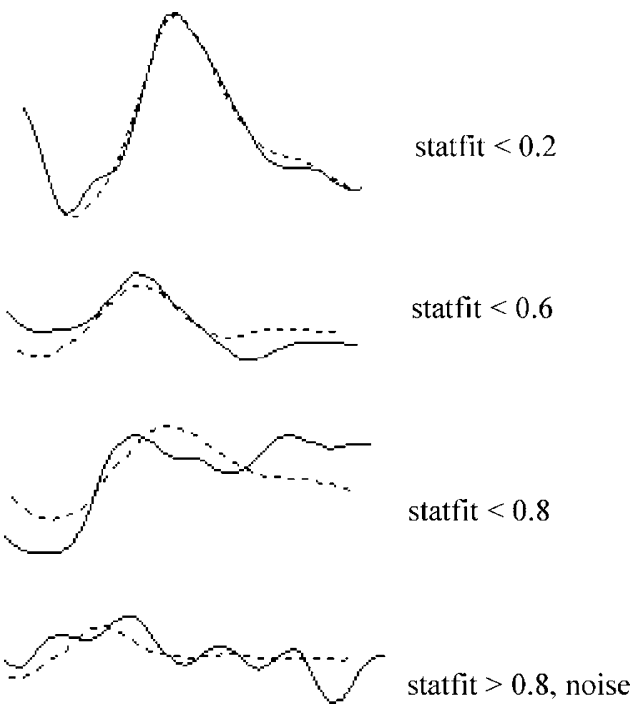

Figure 4 ARM subjects' local responses (solid lines) were fitted against templates for each location (dashed lines) derived from the normal age-matched controls. A goodness of fit cutoff of 0.8 was chosen. Statfit values greater than 0.8 were considered as noise and discarded.

variance (ANOVA) and post hoc tests (LSD) were conducted to compare the effect of retinal location (CP and SI methods) and subject groups and to investigate if the groups had different effects at the different retinal locations.

\section{Repeatability of rod-mediated mfERG data}

To show the reliability of the rod-mediated mfERG responses, we compared the local amplitudes and latencies of responses of an untrained normal control at baseline with repeated responses taken $20 \mathrm{~min}$ after the first recording, analysed by repeated measures ANOVA. Figure 5 shows the two hexagonal trace arrays and the overall responses (superimposed large waveforms) of the baseline results (bold lines) and the repeated results (dashed lines). Some drift of waveform responses was evident because of the inexperience of the subject and the low cutoff filter value chosen $(1 \mathrm{~Hz})$. Choosing a higher cutoff $(3 \mathrm{~Hz}){ }^{41}$ which may eliminate drift, would also decrease signal amplitude. ${ }^{41}$ For the short-term repeatability results shown in Figure 5, local latencies did not differ significantly $\left(\mathrm{F}_{(2,40)}=0.4, P=0.52\right)$, while the mean amplitudes showed a $13 \%$ decrease $\left(\mathrm{F}_{(2,40)}=6.2\right.$, $P=0.02)$.

We also compared the mean local amplitude and latency results of 10 of our (untrained) control subjects at baseline and 1 year later (Figure 6). Again, the local latency results showed no significant differences $\left(F_{(2,47)}=0.03, P=0.87\right)$ between the two measurements, 


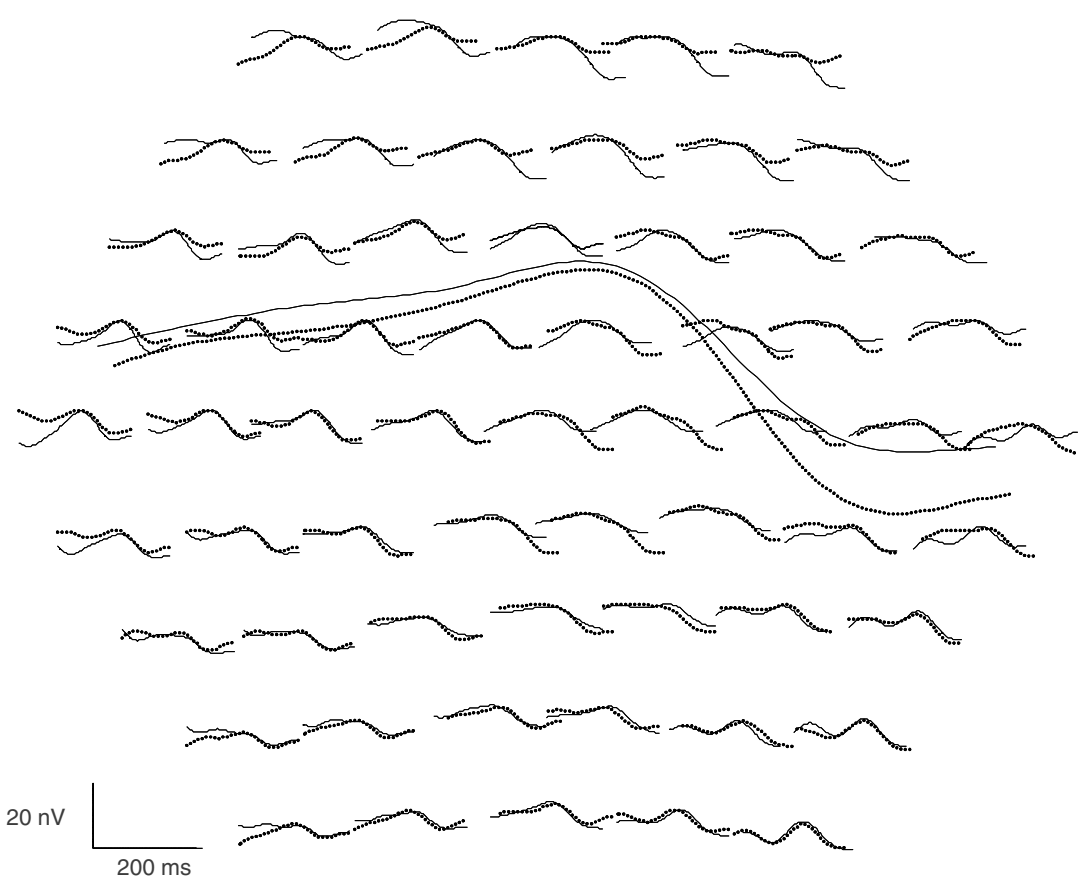

Figure 5 Hexagonal trace array (small waveforms) and the overall response (superimposed large waveform) of the rod-mediated mfERG of a control subject at baseline (bold lines) and repeated 20 min later (dashed lines).

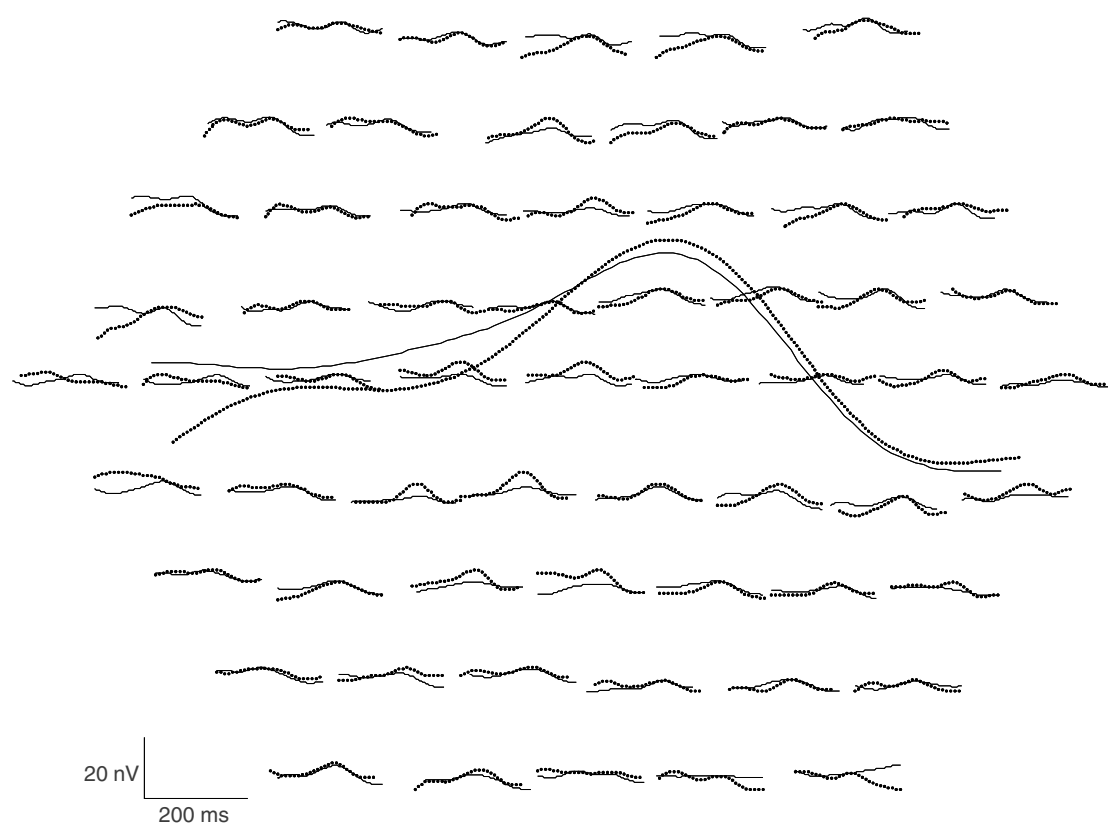

Figure 6 Trace array of the averaged rod-mediated mfERG responses from each hexagon (small waveforms) and the overall response (superimposed larger waveform) of 10 control subjects at baseline (bold lines) and after 1 year (dashed lines). Although there are slightly greater amplitudes in the repeat measures, the peak latencies show stable measures.

suggesting that it is a robust and relatively stable measure. We found an increased mean amplitude by about $28 \%\left(\mathrm{~F}_{(2,47)}=24.6, P<0.01\right)$, which may be due to stray light influence, which is thought to be greater in an older population according to Hood et al. ${ }^{27}$ The scatter of increased lens opacities 1 year later together with the dilated pupil (larger than $8 \mathrm{~mm}$ ) probably caused such an amplitude increase. 


\section{Results}

Significantly delayed central and inferior mean latencies were found for the rods in the ARM1 group compared to the control group (Tables 3 and 4). There was a similar trend for the ARM2 group, but this did not reach statistical significance $(P=0.07)$. Although the cones showed a trend of lower mean a-scales for both ARM groups for both methods, this was not statistically significant (Table 3).

Figure 7 shows the trace arrays for the cones (left) and rods (right) of one subject (subject $h$ ). The cone responses are within the normal range but show some mis-shapen waveforms, while the rods show impaired results centrally, paracentrally, and in the inferior field.

Repeated measures ANOVA of the mean a- and $\mathrm{t}$-scales for the cones (Table 5) showed no statistically significant difference between ARM groups compared to the control group in either CP or SI methods (group effect). However, the mean $\mathrm{t}$-scales for the rods (Table 4, Figure 8) of the ARM1 group were significantly delayed compared to the control group in both methods (CP:

$\mathrm{F}_{2,24}=4.88, P=0.02$; ARM1: post hoc, $P<0.01$; SI:

$\mathrm{F}_{2,24}=4.91, P=0.02$, post hoc, $P<0.01$ ).

Neither the ARM1 nor ARM2 groups' cone or rod responses had amplitude or latency estimates by location that were different from those of the controls, but there

Table 3 Means and standard deviations (SD) for the a- and t-scale results for the central-peripheral (CP) and superior-inferior (SI) methods for the cones and rods of the control and ARM subjects

\begin{tabular}{|c|c|c|c|c|}
\hline Subjects (number of eyes) & Central mean (SD) & Peripheral $(C P)$ mean (SD) & Superior mean (SD) & Inferior (SI) mean (SD) \\
\hline \multicolumn{5}{|l|}{ Cones control (16) } \\
\hline a-scale & $1.05(0.35)$ & $0.99(0.37)$ & $0.98(0.46)$ & $1.02(0.36)$ \\
\hline t-scale & $1.00(0.07)$ & $1.01(0.08)$ & $0.99(0.08)$ & $0.99(0.07)$ \\
\hline \multicolumn{5}{|l|}{ Rods control (13) } \\
\hline a-scale & $1.61(0.37)$ & $1.8(0.27)$ & $1.78(0.38)$ & $1.77(0.24)$ \\
\hline $\mathrm{t}$-scale & $1.18(0.19)$ & $1.12(0.13)$ & $1.07(0.09)$ & $1.16(0.16)$ \\
\hline \multicolumn{5}{|l|}{ Cones ARM1 (11) } \\
\hline a-scale & $0.84(0.29)$ & $0.89(0.22)$ & $0.85(0.31)$ & $0.91(0.25)$ \\
\hline t-scale & $0.94(0.14)$ & $0.98(0.08)$ & $0.95(0.12)$ & $1.0(0.09)$ \\
\hline \multicolumn{5}{|l|}{ Rods ARM1 (9) } \\
\hline a-scale & $1.6(0.36)$ & $1.73(0.33)$ & $1.77(0.37)$ & $1.59(0.22)$ \\
\hline $\mathrm{t}$-scale & $1.43(0.31)^{*}$ & $1.20(0.20)$ & $1.19(0.16)$ & $1.44(0.38)^{*}$ \\
\hline \multicolumn{5}{|l|}{ Cones ARM2 (6) } \\
\hline a-scale & $0.88(0.17)$ & $0.82(0.35)$ & $0.83(0.31)$ & $0.88(0.25)$ \\
\hline t-scale & $0.96(0.08)$ & $1.03(0.12)$ & $0.99(0.11)$ & $1.01(0.10)$ \\
\hline \multicolumn{5}{|l|}{ Rods ARM2 (5) } \\
\hline a-scale & $1.74(0.21)$ & $1.67(0.43)$ & $1.56(0.24)$ & $1.56(0.24)$ \\
\hline t-scale & $1.36(0.16)$ & $1.19(0.11)$ & $1.13(0.10)$ & $1.33(0.32)$ \\
\hline
\end{tabular}

${ }^{*} P<0.01$ statistically significant in comparison to the control group.

Table 4 Rod results of the repeated measures ANOVA for the CP and SI methods

\begin{tabular}{|c|c|c|c|c|}
\hline & & CP method & & SI method \\
\hline \multicolumn{5}{|l|}{$a$-scale } \\
\hline Group effect & $\mathrm{F}_{2,24}=0.01$ & $P=0.99$ & $\mathrm{~F}_{2,24}=0.09$ & $P=0.55$ \\
\hline Group $\times$ location & $\mathrm{F}_{2,24}=0.51$ & $P=0.61$ & $\mathrm{~F}_{2,24}=1.23$ & $P=0.31$ \\
\hline Location effect & $\mathrm{F}_{2,24}=0.42$ & $P=0.52$ & $\mathrm{~F}_{2,24}=0.03$ & $P=0.86$ \\
\hline \multicolumn{5}{|l|}{$t$-scale } \\
\hline Group effect & $\mathrm{F}_{2,24}=4.88$ & $\begin{array}{l}P=0.02 *(\text { between ARM1 and } \\
\left.\mathrm{N} \text { group, post hoc } P<0.01^{*}\right) \\
\text { (between ARM2 and } \\
\mathrm{N} \text { group, post hoc } P=0.07 \text { ) }\end{array}$ & $\mathrm{F}_{2,24}=4.91$ & $\begin{array}{l}P=0.02^{*} \text { (between } \mathrm{ARM} 1 \text { and } \\
\mathrm{N} \text { group, post hoc } P<0.01^{*} \text { ) }\end{array}$ \\
\hline Group $\times$ location & $\mathrm{F}_{2,24}=0.87$ & $P=0.43$ & $\mathrm{~F}_{2,24}=0.79$ & $P=0.47$ \\
\hline Location effect & $\mathrm{F}_{2,24}=6.57$ & $P=0.02^{*}$ (all groups) & $\mathrm{F}_{2,24}=7.82$ & $P=0.01^{*}$ (all groups) \\
\hline
\end{tabular}

*Statistically significant. 
was a significant location effect for the mean of the t-scales for the three groups (Tables 4 and 5). Shorter latencies were found centrally than peripherally for the cones $\left(F_{2,30}=6.47, P=0.02\right)$ and there were longer central (CP: $F_{2,24}=6.57, P=0.02$ ) and inferior latencies (SI: $\left.\mathrm{F}_{2,24}=7.82, P=0.01\right)$ compared to peripheral and superior latencies for the rods for the three groups (Figure 8).

\section{Discussion}

We investigated cone and rod functions in early ARM with the mfERG by using a computerized curve fitting method. Our data indicate a functional impairment of the rods, which is consistent with Curcio's histopathological findings in early ARM. ${ }^{4}$ We found significantly delayed rod latencies in the ARM1 group in both methods. Although there was a trend towards longer mean rod latencies in the ARM2 group compared to the control group, this was not statistically significant. Thus, we could not discriminate between the different levels of early ARM with the rod-mediated mfERG.

In all groups, the mean rod latencies were significantly delayed centrally (central averaged hexagons up to $3 \mathrm{~mm}$ ) compared to peripherally (Table 4), which is in accordance with histopathological findings in ageing and early ARM. ${ }^{1,4}$ Curcio et al. ${ }^{4}$ detected a paracentral rod loss (0.5-2 $\mathrm{mm}$ from the fovea) in ageing and a rod loss particularly $0.5-1 \mathrm{~mm}$ from the foveal centre in early ARM. In contrast to the findings of Curcio et al., ${ }^{1}$ we did not find a predeliction for the lower retina reflecting the
Rods t-scales

CP and SI methods

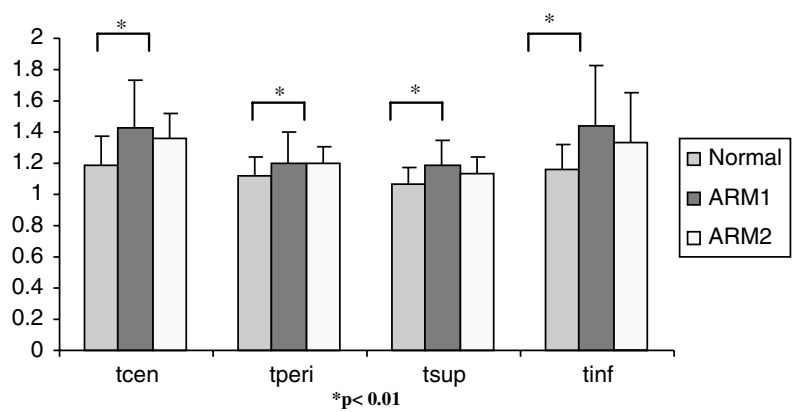

Figure 8 The mean and SD for the $t$-values (note that this value is a ratio) for the rods showed significantly delayed latencies for the ARM1 group compared to the control group. There was also a trend to longer latencies for the ARM2 group, which was not significant. For all groups, latencies were longer centrally and inferiorly compared to the peripheral and superior field.
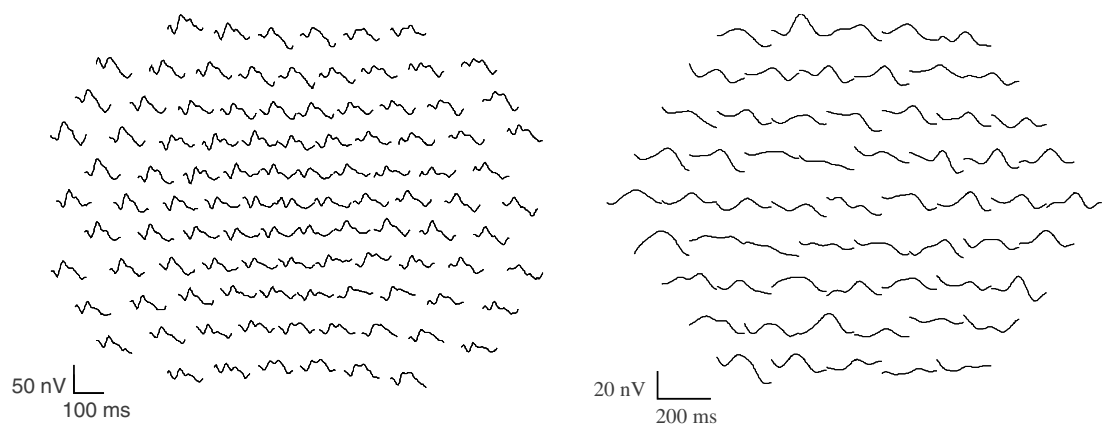

Figure 7 Cone- (left) and rod-mediated (right) mfERG trace arrays from one ARM subject (subject h) right eye. Impaired results are seen for the rods centrally, paracentrally, and inferiorly, while the cone results are mis-shapen but within the normal range. Note the different scaling for the cone- and rod-mediated traces. Drift is evident because of the $1 \mathrm{~Hz}$ lower bandpass cutoff used in the recording.

Table 5 Cone results of the repeated measures ANOVA for the CP and SI methods

\begin{tabular}{|c|c|c|c|c|}
\hline & \multicolumn{2}{|c|}{ CP method } & \multicolumn{2}{|c|}{ SI method } \\
\hline \multicolumn{5}{|l|}{$a$-scale } \\
\hline Group effect & $\mathrm{F}_{2,30}=1.15$ & $P=0.33$ & $\mathrm{~F}_{2,30}=0.14$ & $P=0.55$ \\
\hline Group $\times$ location & $\mathrm{F}_{2,30}=1.86$ & $P=0.17$ & $\mathrm{~F}_{2,30}=0.07$ & $P=0.93$ \\
\hline Location effect & $\mathrm{F}_{2,30}=0.88$ & $P=0.36$ & $\mathrm{~F}_{2,30}=1.68$ & $P=0.21$ \\
\hline \multicolumn{5}{|l|}{ t-scale } \\
\hline Group effect & $\mathrm{F}_{2,30}=0.88$ & $P=0.42$ & $\mathrm{~F}_{2,30}=0.19$ & $P=0.82$ \\
\hline Group $\times$ location & $\mathrm{F}_{2,30}=1.39$ & $P=0.26$ & $\mathrm{~F}_{2,30}=0.84$ & $P=0.44$ \\
\hline Location effect & $\mathrm{F}_{2,30}=6.47$ & $P=0.02^{*}$ (all groups $)$ & $\mathrm{F}_{2,30}=2.79$ & $P=0.11$ \\
\hline
\end{tabular}

*Statistically significant. 
beginning rod impairment, but we found more compromised retinal function in the upper retina (inferior field) for all groups (Table 4). This might be due to the older ARM subjects (on average 70 years) we chose, as Curcio et al. ${ }^{1}$ described their early findings in a younger age group (between 44 and 58 years). Further, this could reflect a beginning rod impairment in our subjects as Curcio et al. ${ }^{1}$ found the highest rod density 4-5 $\mathrm{mm}$ above the fovea in their healthy eyes.

For the cones, all three groups showed significantly shorter mean latencies centrally than peripherally (Table 5). This was more evident, but not statistically significant, for the ARM groups. Seeliger et al. ${ }^{42}$ investigated the implicit time topography of mfERG in subjects with normal vision and fundi and in subjects with retinitis pigmentosa. They found longer latencies centrally compared with the more peripheral retinal areas in their normal group by using a 61 hexagon stimulus and the concentric averaging method. They suggested that retinal asymmetry is evident due to physiological differences, which are presumably at the level of cones. Seeliger et al. ${ }^{42}$ further suggested that it was important to be aware of normal implicit time topography, especially at the onset of maculopathy. If our finding of shorter mean central latencies reflects a beginning functional impairment for the cones, then monitoring latencies across the retina might help in estimating the progression of early ARM. Previous authors ${ }^{23}$ found reduced cone-mediated amplitudes and delayed latencies in early ARM by choosing concentric ring averaging methods. Li et al. ${ }^{23}$ described their ARM subjects as having early macular drusen or irregular pigmentation with RPE changes and a visual acuity of $6 / 8.5$ or better. Although we found significantly faster latencies for all rings for the cones in the ARM1 group by applying their method of averaging the responses to six concentric rings, we found no amplitude reduction compared to the control group using the same method (data not shown). These findings are probably due to different ARM levels and age in their subjects compared to ours. However, in agreement with $\mathrm{Li}$ et al., ${ }^{23}$ we found no statistically significant differences between the superior and inferior fields mean amplitude and latency results.

Hood et al. ${ }^{27}$ had noted in their studies with the rodmediated mfERG that the stimulus and surround conditions that best isolated rod records still needed to be determined and may depend on the particular setup or even the patient population. They suggested that control experiments needed to be performed especially in older populations due to a possible greater stray light influence. We included the suggested stimulus conditions of Hood et al..$^{27}$ and adjusted them to our laboratory conditions and our older subjects including several surround and luminance control experiments. We slowed the stimulation sequence by inserting three blank frames, which has been suggested for clinical use. ${ }^{27}$ Although the amplitude becomes greater as the number of blank frames is increased, the signal-to-noise ratio with three frames has been shown to be as good as with 14 frames inserted. ${ }^{27}$ We overcame the stray light problems by adjusting surround luminance and by lowering the luminance of the stimuli. As reported previously, ${ }^{27}$ we found larger peripheral responses due to stray light when no surround was added (Figure 2). Unlike Hood et al., ${ }^{27}$ who used Burian-Allen electrodes, we used DTL electrodes, which gave poorer signal-tonoise ratio ${ }^{43}$ but more comfort to our subjects. One of the major problems for our subjects was to overcome eye drift and maintain concentration and fixation especially in the rod protocol in which vision was indistinct. Besides breaking up the recording (32 segments), we also used a fixation cross extending to each corner of the screen, which considerably aided fixation. Despite applying Hood et al's. ${ }^{27}$ suggested clinical protocol and the attempt to make it easier for the subjects (DTL electrodes, larger fixation cross, and shorter examination time), many of our subjects still had difficulty controlling eye movements, resulting in more waveform drifts. Thus, the rod-mediated response quality was worse than that of the cone records, a fact that was also found by Hood et al. ${ }^{27}$ It therefore still remains a demanding procedure that might not be suitable for every subject. However, we were able to show that the local rod latency did not change significantly during either short- or long-term follow-up in our control experiments. This demonstrates the reliability of our findings in ARM subjects in which only the latencies were significantly prolonged. The local rod-mediated mfERG amplitudes were less stable and showed more variation. Similar findings have been demonstrated for the local amplitudes of the conemediated mfERG by Fortune et al. ${ }^{39}$ They showed that local cone-mediated mfERG responses differed in amplitude by a factor of about 10 between eyes and about 5 within eyes in normal subjects, whereas the variability of the latency was small suggesting it to be highly reliable.

The reason that the rods are first affected in ARM is unclear. Recent human and animal studies in ARM eyes have found a loss of photoreceptors, RPE cells, and inner nuclear layer cells by apoptosis, preferentially affecting the rods. ${ }^{4-47}$ Curcio et al. ${ }^{3,4}$ hypothesized a vitamin A deficiency and therefore a retinoid deficiency, resulting from a reduced translocation of the retinoids from the blood across Bruch's membrane due to the accumulated cell debris between Bruch's membrane and the RPE. Another reason for preferential rod rather than cone vulnerability might be their larger oxygen requirements; ${ }^{48}$ 
a reduced choroidal blood flow has been demonstrated in ARM. ${ }^{49-51}$ Grunwald et al. ${ }^{49}$ found abnormal choroidal circulation in early stages of ARM and suggested that ischaemia plays a major role in the development of AMD. Friedman et al. ${ }^{50}$ demonstrated increased vascular resistance of the short posterior ciliary arteries in AMD and proposed this to be the result of decreased compliance and calibre of choroidal vessels. An association between reduced rod responses and hypoxia has been described in diabetes. ${ }^{48}$ It is known that ischaemia plays an important role in the pathogenesis and progression of proliferative diabetic retinopathy. ${ }^{52}$ Dark adaptation studies in subjects with early diabetes have also shown effects on the rods. ${ }^{53}$ Additionally, mfERG studies in subjects with and without diabetic retinopathy demonstrated reduced oscillatory potentials, ${ }^{54,55}$ which are thought to be largely rod mediated. ${ }^{56}$ Kurtenbach et al. ${ }^{54}$ showed delayed mfERG oscillatory potentials rather than amplitude reduction in their subjects without diabetic retinopathy. Although we used a different protocol in deriving our rod-mediated mfERGs, we hypothesize that our findings of delayed responses might reflect hypoxia due to reduced blood flow. This might also cause a decrease in the supply of important metabolites (such as vitamin A) to the rod outer segments as Curcio et al..$^{3}$ suggested in their hypothesis.

We were able to record the rod-mediated mfERG in early ARM subjects and to show a functional loss of rod response with the mfERG in early ARM, which has not been described before. This might represent an earlier functional impairment for the rods than for the cones, but this needs to be confirmed by a longitudinal study of a larger sample of older eyes. Our results suggest that a follow-up of rod as well as cone function in older eyes may be predictive of early ARM and may assist in assessing progression or monitoring the effect of treatment for ARM.

\section{Acknowledgements}

We thank Dion Scott for helping to develop the statfit analysis using Matlab (Mathworks, Natick, MA, USA).

\section{References}

1 Curcio C, Millican L, Allen K, Kalina R. Aging of the human photoreceptor mosaic: evidence for selective vulnerability of rods in central retina. Invest Ophthalmol Vis Sci 1993; 34: 3278-3296

2 Curcio C, Medeiros N, Millican L. Photoreceptor loss in agerelated macular degeneration. Invest Ophthalmol Vis Sci 1996; 37: 1236-1249.

3 Curcio C, Owsley C, Jackson G. Spare the rods, save the cones in aging and age-related maculopathy. Invest Ophthalmol Vis Sci 2000; 41: 2015-2018.

4 Curcio C. Photoreceptor topography in aging and agerelated maculopathy. Eye 2001; 15: 376-383.
5 Gartner S, Henkind P. Aging and degeneration of the human macula. 1. Outer nuclear layer and photoreceptors. Br J Ophthalmol 1981; 65: 23-28.

6 Marshall J, Grindle J, Ansell P, Borwien B. Convolution in human rods. Ophthalmology 1979; 63: 181-187.

7 Holopigian K, Seiple W, Greenstein V, Kim D, Carr R. Relative effects of aging and age-related macular degeneration on peripheral visual function. Optom Vis Sci 1997; 74: 152-159.

8 Owsley C, Jackson G, Cideciyan A, Huang Y, Fine S, Ho A et al. Psychophysical evidence for the rod vulnerability in age-related macular degeneration. Invest Ophthalmol Vis Sci 2000; 41: 267-273.

9 Owsley C, Jackson G, White M, Edwards D. Delays in rodmediated dark-adaptation in early age-related maculopathy. Ophthalmology 2001; 108: 1196-1202.

10 Sunness J, Massof R. Focal electro-oculogram in age-related macular degeneration. Am J Optom Physiol Opt 1986; 63: 7-11.

11 Scholl H, Bellmann C, Dandekar S, Bird A, Fitzke F. Photopic and scotopic fine matrix mapping of retinal areas of increased fundus autofluorescence in patients with agerelated maculopathy. Invest Ophthalmol Vis Sci 2004; 45: 574-583.

12 Sutter E, Tran D. The field topography of ERG components in man - I. The photopic luminance response. Vision Res 1992; 32: 433-446.

13 Palmowski A, Sutter E, Bearse M, Fung W. Mapping of retinal function in diabetic retinopathy using the multifocal electroretinogram. Invest Ophthalmol Vis Sci 1997; 38: 2586-2596.

14 Palmowski A, Allgayer R, Heinemann-Vernaleken B, Ruprecht K. First and second order changes in the multifocal electroretinogram of patients with different forms of age related macular degeneration. Vis Sci Appl OSA Tech Dig 2001; 32-35.

15 Palmowski A, Allgayer R, Heinemann-Vernaleken B, Ruprecht K. Influence of photodynamic therapy in choroidal neovascularization on focal retinal function assessed with the multifocal electroretinogram and perimetry. Ophthalmology 2002; 109: 1788-1792.

16 Kretschmann U, Seeliger M, Ruether K, Usui T, ApfelstedtSylla E, Zrenner E. Multifocal electroretinography in patients with Stargardt's macular dystrophy. Br J Ophthalmol 1998; 82: 267-275.

17 Seeliger M, Kretschmann U, Apfelstedt-Sylla E, Ruether K, Zrenner E. Multifocal electroretinography in retinitis pigmentosa. Am J Ophthalmol 1998; 28: 214-226.

18 Hood D, Seiple W, Holopigian K, Greenstein V. A comparison of the components of the multifocal and fullfield ERGs. Vis Neurosci 1997; 14: 533-544.

19 Hood D, Frishman L, Saszik S, Viswanathan S. Retinal origins of the primate multifocal ERG. Implication for the human response. Invest Ophthalmol Vis Sci 2002; 43: 1673-1685.

20 Jackson G, De Leon Ortega J, Girkin C, Rosenstil C, Owsley C. Aging-related changes in the multifocal electroretinogram. Opt Soc Am 2002; 19: 185-189.

21 Seiple W, Vajaranant T, Szlyk J, Clemens C, Holopigian K, Paliga J et al. Multifocal electroretinography as a function of age: the importance of normative values for older adults. Invest Ophthalmol Vis Sci 2003; 44: 1783-1792.

22 Gerth C, Garcia S, Ma L, Keltner J, Werner J. Multifocal electroretinogram: age-related changes for different 
luminance levels. Graefe's Arch Clin Exp Ophthalmol 2002; 240: 202-208.

$23 \mathrm{Li} \mathrm{J}$, Tso M, Lam T. Reduced amplitude and delayed latency in foveal response of multifocal electroretinogram in early age related macular degeneration. Br J Ophthalmol 2001; 85: 287-290.

24 Huang S, Wu D, Jiang F, Ma J, Wu L, Liang J et al. The multifocal electroretinogram in age-related maculopathies. Doc Ophthalmol 2000; 101: 115-124.

25 Jurklies B, Weismann M, Bornfeld N. Multifocal ERG in agerelated macular degeneration with hard and soft drusen. Invest Ophthalmol Vis Sci 2000; 41, ARVO Abstract 4749.

26 Martinson G, Haegerstrom-Portnoy G, Verdon W. Multifocal ERG abnormalities under soft drusen in agerelated maculopathy. Invest Ophthalmol Vis Sci 2000; 42, ARVO Abstract 389.

27 Hood D, Wladis E, Shady S, Holopigian K, Li J, Seiple W. Multifocal rod electroretinograms. Invest Ophthalmol Vis Sci 1998; 39: 1152-1161.

28 Holopigian K, Seiple W, Greenstein V, Hood D, Carr R. Local cone and rod system function in patients with retinitis pigmentosa. Invest Ophthalmol Vis Sci 2001; 42: 779-788.

29 Holopigian K, Seiple W, Greenstein V, Hood D, Carr R. Local cone and rod system function in progressive cone dystrophy. Invest Ophthalmol Vis Sci 2002; 43: 2364-2373.

30 The Age- Related Eye Disease Study Research Group. The age-related eye disease study system for classifying cataracts from photographs: AREDS report number 4. Am J Ophthalmol 2001; 131: 167-175.

31 The Age-Related Eye Disease Study Research Group. The age-related eye disease study system for classifying agerelated macular degeneration from stereoscopic color fundus photographs: the age-related eye disease study report number 6. Am J Ophthalmol 2001; 132: 668-681.

32 Johnson P, Lewis G, Talaga K, Brown M, Kappel P, Fisher S et al. Drusen-associated degeneration in the retina. Invest Ophthalmol Vis Sci 2003; 44: 4481-4488.

33 Wang J, Foran S, Smith W, Mitchell P. Risk of age-related macular degeneration in eyes with macular drusen or hyperpigmentation. The Blue Mountains Eye Study Cohort. Arch Ophthalmol 2003; 121: 658-663.

34 Keating D, Parks S, Evans A. Technical aspects of multifocal ERG recording. Doc Ophthalmol 2000; 100: 77-98.

35 Sutter E. Imaging visual function with the multifocal m-sequence technique. Vision Res 2000; 41: 1241-1255.

36 Keating D, Parks S, Smith D, Evans A. The multifocal ERG: unmasked by selective cross-correlation. Vision Res 2002; 42 2959-2968.

37 Bearse M, Sutter E. Imaging localized retinal dysfunction with the multifocal electroretinogram. Opt Soc Am 1996; 13: 634-640.

38 Hood D, Li J. A technique for measuring individual multifocal ERG records. In: Non-invasive Assessment of the Visual System: Trends in Optics and Photonics 1997; 11: 33-41.
39 Fortune B, Schneck M, Adams A. Multifocal electroretinogram delays reveal local retinal dysfunction in early diabetic retinopathy. Invest Ophthalmol Vis Sci 1999; 40: 2638-2651.

40 Hicks D, Sahel JA. The implications of rod-dependent cone survival for basic and clinical research. Invest Ophthalmol Vis Sci 1999; 40: 3071-3074.

41 Keating D, Parks S, Williamson T, Evans A, Jay J, Elliot A. The effect of pupil dilation, retinal blur and filter bandwith on the multi-focal ERG. Invest Ophthalmol Vis Sci 1996; 37(Suppl): 346.

42 Seeliger M, Kretschmann U, Apfelstedt-Sylla E, Zrenner E. Implicit time topography of multifocal electroretinograms. Invest Ophthalmol Vis Sci 1998; 39: 718-723.

43 Esakowitz L, Kriss A, Shawkat F. A comparison of flash electroretinograms recorded from Burian Allen, JET, Cglide, gold foil, DTL and skin electrodes. Eye 1993; 7: 169-171.

44 Dunaief J, Dentchev T, Ying G-S, Milam A. The role of apoptosis in age-related macular degeneration. Arch Ophthalmol 2002; 120: 1435-1442.

45 Del Priore L, Kuo Y-H, Tezel T. Age-related changes in human RPE cell density and apoptosis proportion in situ. Invest Ophthalmol Vis Sci 2002; 43: 3311-3318.

46 Gordon W, Casey D, Lukiw W, Bazan N. DNA damage and repair in light induced photoreceptor degeneration. Invest Ophthalmol Vis Sci 2002; 43: 3511-3521.

47 Hinton D, He S, Lopez P. Apoptosis in surgically excised choroidal neovascular membranes in age-related macular degeneration. Arch Ophthalmol 1998; 116: 203-209.

48 Arden G, Wolf J, Tsang Y. Does dark adaptation exacerbate diabetic retinopathy? Evidence and a linking hypothesis. Vision Res 1997; 38: 1723-1729.

49 Grunwald J, Hariprasad S, DuPont J, Maguire M, Fine S, Brucker A et al. Foveal choroidal blood flow in age-related macular degeneration (AMD). Invest Ophthalmol Vis Sci 1998; 39: 385-390.

50 Friedman E, Krupsky S, Lane A, Oak S, Friedman E, Egan K et al. Ocular blood flow velocity in age-related macular degeneration. Ophthalmology 1995; 102: 640-646.

51 Pauleikhoff D, Chen J, Chisholm I, Bird A. Choroidal perfusion abnormality with age-related Bruch's membrane change. Am J Ophthalmol 1990; 109: 211-217.

52 Ulbig M, Kampik A. Stage-related therapy of diabetic maculopathy. Ophthalmologe 1993; 90: 395-414.

53 Greenstein V, Thomas S, Blaustein H, Koenig K, Carr R. Effects of early diabetic retinopathy on rod system sensitivity. Optom Vis Sci 1993; 37: 1140-1148.

54 Kurtenbach A, Langrova H, Zrenner E. Multifocal oscillatory potentials in type1 diabetes without retinopathy. Invest Ophthalmol Vis Sci 2000; 41: 3234-3241.

55 Onozu H, Yamamoto S. Oscillatory potentials of multifocal electroretinogram retinopathy. Doc Ophthalmol 2003; 106: 327-332.

$56 \mathrm{Wu}$ S, Sutter E. A topographic study of oscillatory potentials in man. Vis Neurosci 1995; 12: 1013-1025. 